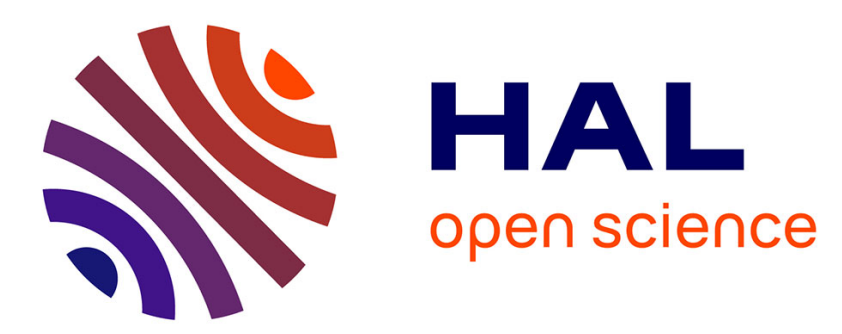

\title{
A nonlinear domain decomposition method to couple compositional gas liquid Darcy and free gas flows
}

Nabil Birgle, Roland Masson, Laurent Trenty

\section{To cite this version:}

Nabil Birgle, Roland Masson, Laurent Trenty. A nonlinear domain decomposition method to couple compositional gas liquid Darcy and free gas flows. FVCA: International Conference on Finite Volumes for Complex Applications VIII, Jun 2017, Lille, France. 10.1007/978-3-319-57397-7 . hal-01541149

\author{
HAL Id: hal-01541149 \\ https://hal.science/hal-01541149
}

Submitted on 25 Jun 2017

HAL is a multi-disciplinary open access archive for the deposit and dissemination of scientific research documents, whether they are published or not. The documents may come from teaching and research institutions in France or abroad, or from public or private research centers.
L'archive ouverte pluridisciplinaire HAL, est destinée au dépôt et à la diffusion de documents scientifiques de niveau recherche, publiés ou non, émanant des établissements d'enseignement et de recherche français ou étrangers, des laboratoires publics ou privés. 


\title{
A nonlinear domain decomposition method to couple compositional gas liquid Darcy and free gas flows
}

\author{
Nabil Birgle* Roland Masson* $\quad$ Laurent Trenty ${ }^{\dagger}$
}

\begin{abstract}
A domain decomposition algorithm is proposed to couple at the interface a gas liquid compositional Darcy flow and a compositional free gas flow. At each time step, our algorithm solves iteratively the nonlinear system coupling the compositional Darcy flow in the porous medium, the RANS gas flow in the free flow domain, and the convection diffusion of the species in the free flow domain. In order to speed up the convergence of the algorithm, the transmission conditions at the interface are replaced by Robin boundary conditions. Each Robin coefficient is obtained from a diagonal approximation of the Dirichlet to Neumann operator related to a scalar simplified model in the neighbouring subdomain. The efficiency of our domain decomposition algorithm is assessed in the case of the modelling of the mass exchanges at the interface between the geological formation and the ventilation galleries of geological radioactive waste disposal.
\end{abstract}

Keywords: Drying model, coupling algorithm, nonlinear domain decomposition method, compositional gas liquid Darcy flow, free gas flow.

\section{Formulation of the coupled model}

Let us denote by $\Omega_{\mathrm{pm}}$ the porous medium domain, by $\Omega_{\mathrm{ff}}$ the free flow domain and by $\Gamma=$ $\partial \Omega_{\mathrm{pm}} \cap \partial \Omega_{\mathrm{ff}}$ the interface. Let $\mathcal{P}=\{g, \ell\}$ denote the set of gas and liquid phases assumed to be both defined by a mixture of components $i \in \mathcal{C}$ among which the water component denoted by $w$ which can vaporize in the gas phase, and a set of gaseous components $j \in$ $\mathcal{C} \backslash\{w\}$ which can dissolve in the liquid phase. The model is assumed to be isothermal with a fixed temperature $T$.

\subsection{Compositional Darcy flow in the porous medium $\Omega_{\mathrm{pm}}$}

Following [1], the liquid gas Darcy flow formulation uses the gas pressure $p^{g}$, the liquid pressure $p^{\ell}$, and the component fugacities $f=\left(f_{i}\right)_{i \in \mathcal{C}}$ as primary unknowns. In this formulation, following [2], the component molar fractions $c^{\alpha}=\left(c_{i}^{\alpha}\right)_{i \in \mathcal{C}}$ of each phase $\alpha \in \mathcal{P}$ are the functions $c^{\alpha}\left(p^{\alpha}, f\right)$ defined by inversion of the equations $f_{i}^{\alpha}\left(c^{\alpha}, p^{\alpha}\right)=f_{i}, i \in \mathcal{C}$, where $f_{i}^{\alpha}$ is the fugacity of the component $i$ in the phase $\alpha$. In addition, for $\alpha \in \mathcal{P}$, the phase pressure $p^{\alpha}$ is extended in the absence of the phase in such a way that the closure law

*Université Côte d'Azur, Inria, CNRS, Laboratoire J.A. Dieudonné, Team Coffee, Parc Valrose, 06108 Nice cedex 02, France. $\dagger$ Andra,

1-7 rue Jean Monnet 92290 Chatenay-Malabry, France. 
$\sum_{i \in \mathcal{C}} c_{i}^{\alpha}\left(p^{\alpha}, f\right)=1$ is always imposed (see [1]). The phase molar and mass densities, as well as the phase viscosities functions are denoted in the following by respectively $\zeta^{\alpha}\left(p^{\alpha}, c^{\alpha}\right)$, $\rho^{\alpha}\left(p^{\alpha}, c^{\alpha}\right), \mu^{\alpha}\left(p^{\alpha}, c^{\alpha}\right)$ for $\alpha \in \mathcal{P}$. Finally, we define the liquid saturation as the function $s^{\ell}\left(\mathbf{x}, p^{g}-p^{\ell}\right)$ defined by the inverse of the monotone graph extension of the capillary pressure function, and we set $s^{g}(\mathbf{x},)=.1-s^{\ell}(\mathbf{x},$.$) .$

Let us define the two-phase Darcy velocities $\mathbf{u}^{\alpha}=\frac{-k_{r}^{\alpha}}{\mu^{\alpha}} \mathbf{K}\left(\nabla p^{\alpha}-\rho^{\alpha} \mathbf{g}\right)$ where $k_{r}^{\alpha}\left(\mathbf{x}, s^{\alpha}\right)$ is the phase relative permeability, $\mathbf{K}(\mathbf{x})$ the porous medium permeability tensor, and $\mathbf{g}$ the gravitational acceleration vector. Let us also introduce, for each component $i \in \mathcal{C}$, the total number of mole per unit pore volume $\mathrm{n}_{i}=\sum_{\alpha \in \mathcal{P}} s^{\alpha} \zeta^{\alpha} c_{i}^{\alpha}$ and the component molar flow rate $\mathbf{v}_{i}=\sum_{\alpha \in \mathcal{P}} \zeta^{\alpha} c_{i}^{\alpha} \mathbf{u}^{\alpha}$. The model in $\Omega_{\mathrm{pm}}$ accounts for the mole conservation of each component $i \in \mathcal{C}$ coupled with the sum to 1 of the molar fractions for each phase $\alpha \in \mathcal{P}$ :

$$
\begin{aligned}
\phi \partial_{t} \mathrm{n}_{i}+\nabla \cdot \mathbf{v}_{i} & =0, i \in \mathcal{C}, & & \text { on } \Omega_{\mathrm{pm}} \times\left(0, t_{\mathrm{f}}\right), \\
\sum_{i} c_{i}^{\alpha} & =1, \alpha \in \mathcal{P}, & & \text { on } \Omega_{\mathrm{pm}} \times\left(0, t_{\mathrm{f}}\right),
\end{aligned}
$$

where $\phi(\mathbf{x})$ is the porous medium porosity and $\left(0, t_{\mathrm{f}}\right)$ the simulation time interval.

\subsection{Flow and transport model in the free flow domain $\Omega_{\mathrm{ff}}$}

The primary unknowns in the free flow domain are defined by the gas pressure $p$, the gas molar fractions $c=\left(c_{i}\right)_{i \in \mathcal{C}}$, and the gas velocity $\mathbf{u}$. The flow is described by a Reynolds Averaged Navier-Stokes (RANS) model and assumed to be quasi-stationary at the time scale of the porous medium. Let us first define the uncoupled mean turbulent flow as the solution $\left(\mathbf{u}^{0}, p^{0}\right)$ of the following RANS model given the initial gas molar fractions $c^{0}$ :

$$
\begin{aligned}
\nabla \cdot\left(\rho^{g}\left(p^{0}, c^{0}\right) \mathbf{u}^{0} \otimes \mathbf{u}^{0}-\mu_{\mathrm{t}}^{0}\left(\nabla \mathbf{u}^{0}+\nabla^{\mathrm{t}} \mathbf{u}^{0}\right)\right)+\nabla p^{0} & =\rho^{g}\left(p^{0}, c^{0}\right) \mathbf{g}, & & \text { in } \Omega_{\mathrm{ff}}, \\
\nabla \cdot\left(\zeta^{g}\left(p^{0}, c^{0}\right) \mathbf{u}^{0}\right) & =0, & & \text { in } \Omega_{\mathrm{ff}}, \\
\mathbf{u}^{0} & =0, & & \text { on } \Gamma,
\end{aligned}
$$

where the turbulent viscosity $\mu_{\mathrm{t}}^{0}(\mathbf{x})$ is obtained using an algebraic turbulent model or a more advanced $k-\epsilon$ model [3]. This turbulent flow is responsible for a turbulent diffusivity denoted by $d_{\mathrm{t}}^{0}(\mathbf{x})$ typically depending on the turbulent viscosity, on the gas Fickian diffusion and on the Schmidt number. Assuming that the velocity perturbation induced by the coupling is small compared to the flow velocity, the turbulent viscosity $\mu_{\mathrm{t}}^{0}$ and diffusivity $d_{\mathrm{t}}^{0}$ are used for the coupled model. Thus, the primary unknowns $\mathbf{u}, p, c$ in the free flow domain satisfy the following system of equations

$$
\begin{aligned}
\nabla \cdot\left(\rho^{g}(p, c) \mathbf{u} \otimes \mathbf{u}-\mu_{\mathrm{t}}^{0}\left(\nabla \mathbf{u}+\nabla^{\mathrm{t}} \mathbf{u}\right)\right)+\nabla p & =\rho^{g}(p, c) \mathbf{g}, & & \text { on } \Omega_{\mathrm{ff}} \times\left(0, t_{\mathrm{f}}\right), \\
\nabla \cdot\left(\zeta^{g}(p, c) \mathbf{u}\right) & =0, & & \text { on } \Omega_{\mathrm{ff}} \times\left(0, t_{\mathrm{f}}\right), \\
\nabla \cdot \mathbf{w}_{i} & =0, i \in \mathcal{C}, & & \text { on } \Omega_{\mathrm{ff}} \times\left(0, t_{\mathrm{f}}\right),
\end{aligned}
$$

with the component molar flow rate $\mathbf{w}_{i}=\zeta^{g}(p, c)\left(c_{i} \mathbf{u}-d_{\mathrm{t}}^{0} \nabla c_{i}\right)$.

\subsection{Transmission conditions at the interface $\Gamma$}

At the interface $\Gamma$ between the free-flow domain and the porous medium, the coupling conditions are those stated in $[4,3,5]$ where we have replaced the Beaver Joseph condition by the simpler no slip condition due to the low permeability of the porous medium in our application. They state the gas molar fraction and molar normal flux continuity, the gas 
liquid thermodynamical equilibrium, the no slip condition, and the normal component of the normal stress continuity as follows:

$$
\begin{aligned}
c_{i}^{g} & =c_{i}, & & \text { on } \Gamma \times\left(0, t_{\mathrm{f}}\right), \\
\mathbf{v}_{i} \cdot \mathbf{n}_{\mathrm{pm}}+\mathbf{w}_{i} \cdot \mathbf{n}_{\mathrm{ff}} & =0, & & \text { on } \Gamma \times\left(0, t_{\mathrm{f}}\right), \\
\sum_{i} c_{i}^{\alpha} & =1, & & \text { on } \Gamma \times\left(0, t_{\mathrm{f}}\right), \\
\left(\rho^{g} \mathbf{u} \otimes \mathbf{u}-\mu_{\mathrm{t}}^{0}\left(\nabla \mathbf{u}+\nabla^{\mathrm{t}} \mathbf{u}\right)\right) \mathbf{n}_{\mathrm{ff}} \cdot \mathbf{n}_{\mathrm{ff}}+p & =p^{g}, & & \text { on } \Gamma \times\left(0, t_{\mathrm{f}}\right), \\
\mathbf{u} \wedge \mathbf{n}_{\mathrm{ff}} & =0, & & \text { on } \Gamma \times\left(0, t_{\mathrm{f}}\right),
\end{aligned}
$$

with $i \in \mathcal{C}, \alpha \in \mathcal{P}$, and where $\mathbf{n}_{\mathrm{pm}}$ and $\mathbf{n}_{\mathrm{ff}}$ are the unit normal vectors at the interface $\Gamma$ oriented outward from the porous medium domain and the free flow domain respectively.

\section{Domain decomposition algorithm}

The coupled model (1)-(3)-(4) is integrated in time using an Euler implicit scheme which leads to solve at each time step a fully coupled nonlinear system. This nonlinear system is solved using a domain decomposition algorithm detailed below. This approach has two advantages. Firstly it allows to use different codes for the porous medium and the free flow problems. Secondly, it reduces the complexity of the nonlinear and linear systems which results in a better efficiency compared with a monolithic Newton algorithm solving the fully coupled system $[4,3]$.

In the following, the time step count $n$ is omitted for the sake of clarity and the component total number of mole in the porous medium at the previous time step is denoted by $\mathrm{n}_{i}^{n-1}$. The domain decomposition count is denoted by the superscript $k$. As usual, the algorithm is initialized by the previous time step solution. The algorithm solves iteratively, until convergence to the fully coupled solution, the compositional gas liquid flow in the porous medium with Robin type transmission conditions, the RANS model in the free flow domain and the convection diffusion equations in the free flow domain with Robin type transmission conditions.

\subsection{Porous medium flow with Robin boundary conditions on $\Gamma$}

Compute the phase pressures $p^{\alpha, k}, \alpha \in \mathcal{P}$, the fugacity vector $f^{k}$ in the porous medium $\Omega_{\mathrm{pm}}$ and a normal velocity correction denoted by $\delta_{\mathbf{u}}^{k}$ at the interface $\Gamma$ and oriented outward to the free flow domain, such that

$$
\begin{aligned}
\frac{\phi}{\Delta t^{n}}\left(\mathrm{n}_{i}^{k}-\mathrm{n}_{i}^{n-1}\right)+\nabla \cdot \mathbf{v}_{i}^{k}=0, & \text { in } \Omega_{\mathrm{pm}}, \\
\sum_{i} c_{i}^{\alpha, k}=1, & \text { in } \Omega_{\mathrm{pm}}, \\
\beta_{\mathrm{pm}} c_{i}^{g, k}-\mathbf{v}_{i}^{k} \cdot \mathbf{n}_{\mathrm{pm}}-c_{i}^{g, k} \zeta_{\mathrm{pm}}^{g, k} \delta_{\mathbf{u}}^{k}=\beta_{\mathrm{pm}} c_{i}^{k-1}-\mathbf{w}_{i}^{k-1} \cdot \mathbf{n}_{\mathrm{pm}}, & \text { on } \Gamma, \\
p^{g, k}=p^{k-1}+\left(\rho_{\mathrm{ff}}^{g} \mathbf{u} \otimes \mathbf{u}-\mu_{\mathrm{t}}^{0}\left(\nabla \mathbf{u}+\nabla^{\mathrm{t}} \mathbf{u}\right)\right)^{k-1} \mathbf{n}_{\mathrm{ff}} \cdot \mathbf{n}_{\mathrm{ff}}, & \text { on } \Gamma, \\
\sum_{i} c_{i}^{\alpha, k}=1, & \text { on } \Gamma .
\end{aligned}
$$

with $i \in \mathcal{C}, \alpha \in \mathcal{P}$ and $\zeta_{\mathrm{pm}}^{g, k}=\zeta^{g}\left(p^{g, k}, c^{g, k}\right)$. Note that the additional unknown $\delta_{\mathbf{u}}^{k}$ accounts for the correction of the normal gas velocity $\mathbf{u}^{k-1} \cdot \mathbf{n}_{\mathrm{ff}}$ at the interface induced by the coupling with the porous medium. 


\subsection{RANS flow with Dirichlet boundary condition on $\Gamma$}

Compute the pressure $p^{k}$ and the gas velocity $\mathbf{u}^{k}$ such that

$$
\begin{aligned}
\nabla \cdot\left(\rho_{\mathrm{ff}}^{g, k} \mathbf{u}^{k} \otimes \mathbf{u}^{k}-\mu_{\mathrm{t}}^{0}\left(\nabla \mathbf{u}^{k}+\nabla^{t} \mathbf{u}^{k}\right)\right)+\nabla p^{k}=\rho_{\mathrm{ff}}^{g, k} \mathbf{g}, & \text { in } \Omega_{\mathrm{ff}}, \\
\nabla \cdot\left(\zeta_{\mathrm{ff}}^{g, k} \mathbf{u}^{k}\right)=0, & \text { in } \Omega_{\mathrm{ff}}, \\
\zeta_{\mathrm{ff}}^{g, k} \mathbf{u}^{k}=\zeta_{\mathrm{ff}}^{g, k-1} \mathbf{u}^{k-1}+\zeta_{\mathrm{pm}}^{g, k} \delta_{\mathbf{u}}^{k} \mathbf{n}_{\mathrm{ff}}, & \text { on } \Gamma,
\end{aligned}
$$

with $\zeta_{\mathrm{ff}}^{g, k}=\zeta^{g}\left(p^{k}, c^{k-1}\right)$ and $\rho_{\mathrm{ff}}^{g, k}=\rho^{g}\left(p^{k}, c^{k-1}\right)$.

\subsection{Convection diffusion equations with Robin boundary conditions on $\Gamma$}

Compute $c^{k}$ such that for all $i \in \mathcal{C}$

$$
\begin{aligned}
\nabla \cdot \mathbf{w}_{i}^{k}=0, & \text { in } \Omega_{\mathrm{ff}}, \\
\beta_{\mathrm{ff}} c_{i}^{k}-\mathbf{w}_{i}^{k} \cdot \mathbf{n}_{\mathrm{ff}}=\beta_{\mathrm{ff}} c_{i}^{g, k}-\mathbf{v}_{i}^{k} \cdot \mathbf{n}_{\mathrm{ff}}, & \text { on } \Gamma,
\end{aligned}
$$

with $\mathbf{w}_{i}^{k}=\zeta_{\mathrm{ff}}^{g, k}\left(c_{i}^{k} \mathbf{u}^{k}-d_{\mathrm{t}}^{0} \nabla c_{i}^{k}\right)$.

The domain decomposition algorithm is iterated until the following stopping criterion at the interface $\Gamma$ is satisfied for a given tolerance $\epsilon$ :

$$
\frac{\sum_{i \in \mathcal{C}}\left\|c_{i}^{g, k}-c_{i}^{k}\right\|}{\sum_{i \in \mathcal{C}}\left\|c_{i}^{k}\right\|}+\frac{\sum_{i \in \mathcal{C}}\left\|\left(\mathbf{v}_{i}^{k}-\mathbf{w}_{i}^{k}\right) \cdot \mathbf{n}_{\mathrm{ff}}\right\|}{\sum_{i \in \mathcal{C}}\left\|\mathbf{w}_{i}^{k} \cdot \mathbf{n}_{\mathrm{ff}}\right\|}+\frac{\left\|\delta_{\mathbf{u}}^{k}\right\|}{\left\|\mathbf{u}^{k} \cdot \mathbf{n}_{\mathrm{ff}}\right\|}<\varepsilon .
$$

\subsection{Computation of the Robin coefficients $\beta_{\mathrm{pm}}$ and $\beta_{\mathrm{ff}}$}

To speedup the convergence of the domain decomposition method, the Robin coefficients $\beta_{\mathrm{pm}}$ and $\beta_{\mathrm{ff}}$ of each subdomain must approximate the Dirichlet to Neumann (DtN) operator of the neighbouring subdomain problem [6]. For this purpose, a simplified scalar model is defined in each subdomain and a low frequency diagonal approximation of its DtN operator is built.

To compute $\beta_{\mathrm{pm}}$, the convection diffusion equation in (3) is approximated by using the uncoupled velocity $\mathbf{u}^{0}$ from (2) and by neglecting the variations of the gas molar density. We end up with a linear operator $\mathcal{L}_{\mathrm{ff}} c=\zeta^{g}\left(p^{0}, c^{0}\right) \nabla \cdot\left(c \mathbf{u}^{0}-d_{\mathrm{t}}^{0} \nabla c\right)$ independent on $i \in \mathcal{C}$. Thus for a molar fraction $c_{\Gamma}$ on $\Gamma$, we define $\operatorname{DtN}_{\mathrm{ff}}\left(c_{\Gamma}\right)=\mathbf{w} \cdot \mathbf{n}_{\mathrm{ff}}$ where $\mathbf{w}=\zeta^{g}\left(p^{0}, c^{0}\right)\left(c \mathbf{u}^{0}-d_{\mathrm{t}}^{0} \nabla c\right)$ and $c$ is solution of the convection diffusion equation $\nabla \cdot \mathbf{w}=0$ in $\Omega_{\mathrm{ff}}$ with Dirichlet condition $c=c_{\Gamma}$ on $\Gamma$. To account efficiently for the convection diffusion boundary layer and the tangential convection, the following low frequency diagonal approximation of the $\mathrm{DtN}_{\mathrm{ff}}$ operator

$$
\beta_{\mathrm{pm}}=\operatorname{DtN}_{\mathrm{ff}}\left(1_{\Gamma}\right)-\operatorname{DtN}_{\mathrm{ff}}\left(0_{\Gamma}\right)
$$

is used rather than a classical order 0 Taylor approximation.

To compute $\beta_{\mathrm{ff}}$, the gas liquid porous medium flow is approximated by the Richards equation. Let us define $\bar{c}_{i}^{\ell}=1$ for $i=w$ and $\bar{c}_{i}^{\ell}=0$ for $i \in \mathcal{C} \backslash\{w\}$, and let $\bar{p}^{g}$ be a constant reference pressure in the free flow domain typically corresponding to the outflow pressure. From these, the state laws are approximated by $\bar{\zeta}^{\ell}\left(p^{\ell}\right)=\zeta^{\ell}\left(p^{\ell}, \bar{c}^{\ell}\right)$, $\bar{\mu}^{\ell}\left(p^{\ell}\right)=\mu^{\ell}\left(p^{\ell}, \bar{c}^{\ell}\right)$ and $\bar{\rho}^{\ell}\left(p^{\ell}\right)=\rho^{\ell}\left(p^{\ell}, \bar{c}^{\ell}\right)$ and the water molar fraction in the gas is given by $\bar{c}_{w}^{g}\left(p^{\ell}\right)=c_{w}^{g}\left(\bar{p}^{g}, f^{\ell}\left(\bar{c}^{\ell}, p^{\ell}\right)\right)$. Let us set $\mathrm{n}^{\ell}\left(\mathbf{x}, p^{\ell}\right)=\bar{\zeta}^{\ell}\left(p^{\ell}\right) s^{\ell}\left(\mathbf{x}, \bar{p}^{g}-p^{\ell}\right)$ and 


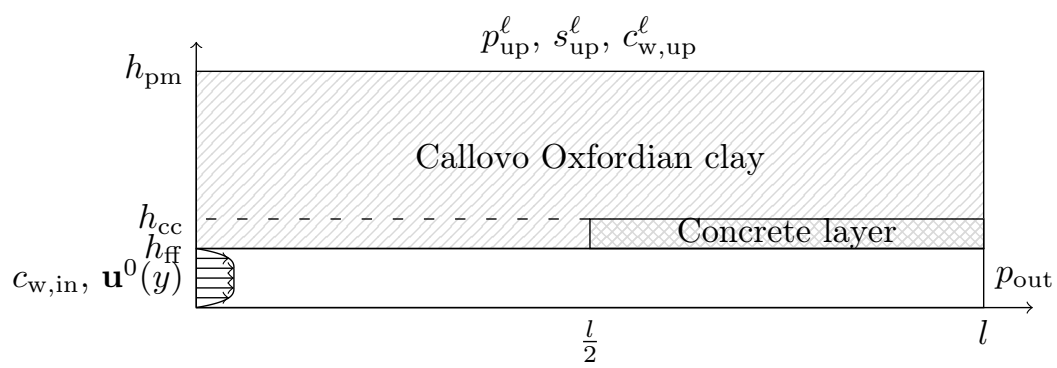

Figure 1 - Computational domain.

$M^{\ell}\left(\mathbf{x}, p^{\ell}\right)=\frac{\bar{\zeta}^{\ell}\left(p^{\ell}\right)}{\bar{\mu}^{\ell}\left(p^{\ell}\right)} k_{r}^{\ell}\left(\mathbf{x}, s^{\ell}\left(\mathbf{x}, \bar{p}^{g}-p^{\ell}\right)\right)$. The Richards model with prescribed water molar fraction $c_{w}$ at $\Gamma$ is defined as follows after time integration using the implicit Euler scheme:

$$
\begin{aligned}
\frac{\phi}{\Delta t^{n}}\left(\mathrm{n}^{\ell}-\mathrm{n}^{\ell, n-1}\right)+\nabla \cdot \mathbf{v}^{\ell} & =0, & & \text { in } \Omega_{\mathrm{pm}}, \\
\bar{c}_{w}^{g}\left(p^{\ell}\right) & =c_{w}, & & \text { on } \Gamma,
\end{aligned}
$$

where $\mathrm{n}^{\ell, n-1}(\mathbf{x})=\mathrm{n}^{\ell}\left(\mathbf{x}, p^{\ell, n-1}\right)$ and $\mathbf{v}^{\ell}=-M^{\ell} \mathbf{K}\left(\nabla p^{\ell}-\bar{\rho}^{\ell} \mathbf{g}\right)$. At each point of the interface $\Gamma$ the equation (9) is linearized with respect to $p^{\ell}$ and its coefficients are freezed leading to $\mathcal{L}_{\mathrm{pm}} \delta p^{\ell}=\eta \delta p^{\ell}+\nabla \cdot\left(-\kappa \nabla \delta p^{\ell}+\psi \delta p^{\ell}\right)$ with the Dirichlet boundary condition $\delta p^{\ell}=\frac{\delta c_{w}}{\partial_{p^{\ell}} \bar{c}_{w}^{g}}$ on $\Gamma$. The freezed coefficients are defined by $\eta=\frac{\phi}{\Delta t^{n}} \partial_{p^{\ell}} \mathrm{n}^{\ell}, \kappa=M^{\ell} \mathbf{K}$ and $\psi=-\partial_{p^{\ell}} M^{\ell} \mathbf{K} \nabla p^{\ell}+$ $\partial_{p^{\ell}}\left(M^{\ell} \bar{\rho}^{\ell}\right) \mathbf{K g}$. The Robin coefficient is obtained using the following DtN order 0 Taylor approximation [6]:

$$
\beta_{\mathrm{ff}}=\frac{1}{2 \partial_{p^{\ell}} \bar{c}_{w}^{g}}\left(\psi \cdot \mathbf{n}_{\mathrm{ff}}+\sqrt{\left(\psi \cdot \mathbf{n}_{\mathrm{ff}}\right)^{2}+4 \eta \kappa \mathbf{n}_{\mathrm{ff}} \cdot \mathbf{n}_{\mathrm{ff}}}\right) .
$$

\section{Numerical experiment}

This test case is a simplified two dimensional setting defined with Andra [5] to simulate the mass exchanges occurring within deep geological radioactive waste disposal at the interface between a geological formation and a ventilation excavated gallery.

The computational domain shown in Fig. 1 is a rectangle of length $l=100 \mathrm{~m}$ and height $h_{\mathrm{pm}}=15 \mathrm{~m}$, split horizontally into the free flow domain $\Omega_{\mathrm{ff}}=(0, l) \times\left(0, h_{\mathrm{ff}}\right)$ of height $h_{\mathrm{ff}}=5 \mathrm{~m}$ and the porous medium $\Omega_{\mathrm{pm}}=(0, l) \times\left(h_{\mathrm{ff}}, h_{\mathrm{pm}}\right)$. The temperature is fixed to $T=303 \mathrm{~K}$ both in the porous medium and the free flow domains. The gas and liquid phases are a mixture of air $(a)$ and water $(w)$ components. The liquid and gas properties are defined by $\zeta^{\ell}=55555$ mol. $\mathrm{m}^{-3}, \mu^{\ell}=10^{-3}$ Pa.s, $\mu^{g}=1.851 \cdot 10^{-5} \mathrm{~Pa} . \mathrm{s}, \zeta^{g}=p^{g}(R T)^{-1}$, $\rho^{\alpha}=\zeta^{\alpha} \sum_{i \in \mathcal{C}} c_{i}^{\alpha} m^{i}, \alpha \in \mathcal{P}$ with the molar masses $m^{w}=18 \cdot 10^{-3} \mathrm{~kg} \cdot \mathrm{mol}^{-1}$ and $m^{a}=$ $29 \cdot 10^{-3} \mathrm{~kg} \cdot \mathrm{mol}^{-1}$. The fugacities are defined in the liquid phase by the Raoult-Kelvin's law for the water component and the Henry's law for the air which leads by inversion to $c_{w}^{\ell}\left(p^{\ell}, f\right)=\frac{f_{w}}{p_{\text {sat }}} e^{\frac{p_{\text {sat }}-p^{\ell}}{\zeta^{\ell} R T}}, c_{a}^{\ell}\left(p^{\ell}, f\right)=\frac{f_{a}}{H^{a}}$, where $p_{\text {sat }}=4138 \mathrm{~Pa}$ at $T=303 \mathrm{~K}$ and $H^{a}=3.33 \cdot 10^{9} \mathrm{~Pa}$. The gas fugacities are defined by the Dalton's law for an ideal mixture of perfect gas leading to $c_{i}^{g}\left(p^{g}, f\right)=\frac{f_{i}}{p^{g}}$.

The porous medium contains two rocktypes: a concrete layer located in $\Omega_{\mathrm{cc}}=\left(\frac{l}{2}, l\right) \times$ $\left(h_{\mathrm{ff}}, h_{\mathrm{cc}}\right)$ with $h_{\mathrm{cc}}=6 \mathrm{~m}$ and the Callovo Oxfordian clay elsewhere in $\Omega_{\mathrm{cox}}=\Omega_{\mathrm{pm}} \backslash \Omega_{\mathrm{cc}}$. 


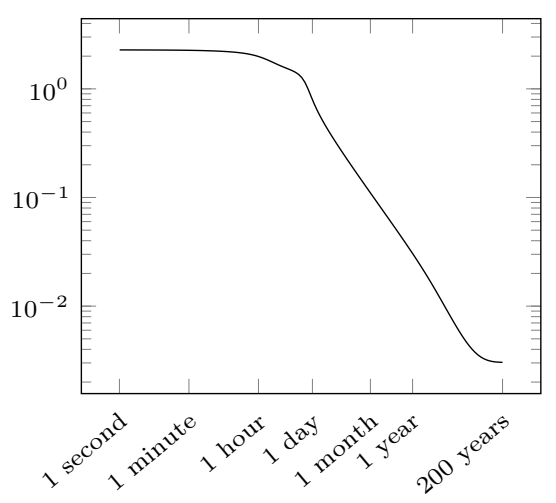

Time

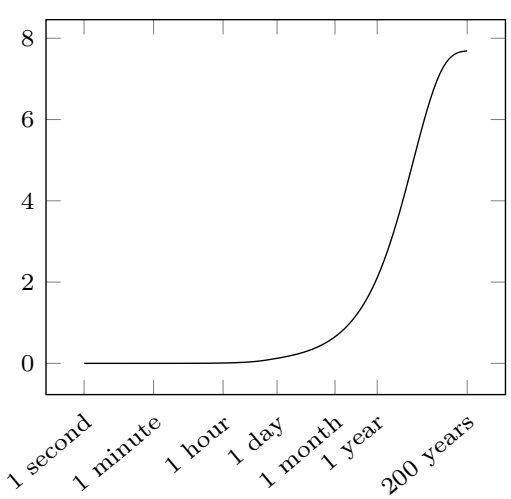

Time

Figure 2 - Evaporation rate at the interface in $1 . \mathrm{d}^{-1} \cdot \mathrm{m}^{-1}$ (left) and gas volume in the porous medium in $\mathrm{m}^{3}$ (right).

The liquid saturation and the relative permeabilities are given by the Van Genuchten laws as in [5] with parameters set to $n_{\mathrm{r}}=1.54, m_{\mathrm{r}}=1-\frac{1}{n_{\mathrm{r}}}, p_{\mathrm{r}}=2 \cdot 10^{6} \mathrm{~Pa}, s_{\mathrm{r}}^{\ell}=0.01, s_{\mathrm{r}}^{g}=0$ for the concrete rocktype and to $n_{\mathrm{r}}=1.49, m_{\mathrm{r}}=1-\frac{1}{n_{\mathrm{r}}}, p_{\mathrm{r}}=15 \cdot 10^{6} \mathrm{~Pa}, s_{\mathrm{r}}^{\ell}=0.4, s_{\mathrm{r}}^{g}=0$ for the Cox rocktype. The porosity is set to $\phi=0.3$ (resp. $\phi=0.15$ ) and the permeability is isotropic and set to $\mathbf{K}=10^{-18} \mathrm{~m}^{2}$ (resp. $\mathbf{K}=5 \cdot 10^{-20} \mathrm{~m}^{2}$ ) in the concrete rocktype (resp. Cox rocktype).

The liquid pressure, the liquid saturation and the water mole fraction are set both at the initial time and at the top of the porous medium $\Gamma_{\mathrm{up}}=(0, l) \times\left\{h_{\mathrm{pm}}\right\}$ to $p^{\ell, 0}=p_{\mathrm{up}}^{\ell}=$ $4 \cdot 10^{6} \mathrm{~Pa}, s^{\ell, 0}=s_{\mathrm{up}}^{\ell}=1$ and $c_{w}^{\ell, 0}=c_{\mathrm{w}, \mathrm{up}}^{\ell}=1$ respectively. In the free flow domain, the mean uncoupled turbulent velocity profile $\mathbf{u}^{0}(y)$ is obtained using the Prandtl algebraic turbulent model [5] which defines the turbulent viscosity $\mu_{\mathrm{t}}^{0}$. The turbulent diffusion $d_{\mathrm{t}}^{0}=d^{g}+\frac{\mu_{\mathrm{t}}^{0}-\mu^{g}}{\rho^{g} S_{\mathrm{c}}}$ is deduced using the gas Fickian diffusion $d^{g}=2 \cdot 10^{-5} \mathrm{~m} \cdot \mathrm{s}^{-1}$ and the Schmidt number $S_{\mathrm{c}}=1$. At the output interface $\Gamma_{\text {out }}=\{l\} \times\left(0, h_{\mathrm{ff}}\right)$, the pressure $p_{\text {out }}=10^{5} \mathrm{~Pa}$ is the atmospheric pressure which also corresponds to the pressure $\bar{p}^{g}$ used to compute the Robin coefficient $\beta_{\mathrm{ff}}^{n}$. The velocity at the input boundary $\Gamma_{\mathrm{in}}=\{0\} \times\left(0, h_{\mathrm{ff}}\right)$ is defined by the velocity profile $\mathbf{u}^{0}(y)$ and is such that $u_{\text {in }}=-\left|\Gamma_{\text {in }}\right|^{-1} \int_{\Gamma_{\mathrm{in}}} \mathbf{u}^{0}(y) \cdot \mathbf{n}_{\mathrm{ff}}=0.5 \mathrm{~m} . \mathrm{s}^{-1}$. The input water molar fraction $c_{\mathrm{w} \text {, in }}$ corresponds to a relative humidity $H_{r}=\frac{p_{\text {out }} c_{\mathrm{w}, \text { in }}}{p_{\mathrm{sat}}}=0.5$. Homogeneous Neumann boundary conditions are used at the others boundaries of the domain.

Following [5], a Cartesian mesh of size $100 \times 242$ refined at interface $\Gamma$ is used. The Darcy problem in (5) and the convection diffusion equation in (7) are solved using a two point flux approximation method given in [7] with additional face unknowns at the interface $\Gamma$. The RANS problem in (6) is solved using a staggered Marker And Cell scheme given in [8]. An implicit Euler scheme is used in time with a time step $\Delta t^{n}=(1.2)^{n-1} \mathrm{~s}$ which varies exponentially up to reach the final simulation time $t_{\mathrm{f}}=200$ years, which corresponds roughly to the time of ventilation of the storage and is large enough to reach the stationary state.

Fig. 2 shows the evaporation rate in the gallery and the gas volume in the porous medium as a function of time. A drop of the evaporation rate occurs at $t \simeq 1$ day when the interface is not longer saturated with water. Fig. 3 exhibits the Robin coefficients obtained for this test case. The dependence on time of $\beta_{\mathrm{ff}}$ is crucial to obtain the convergence of the algorithm and corresponds roughly to a Dirichlet condition before the drop of the evaporation rate 

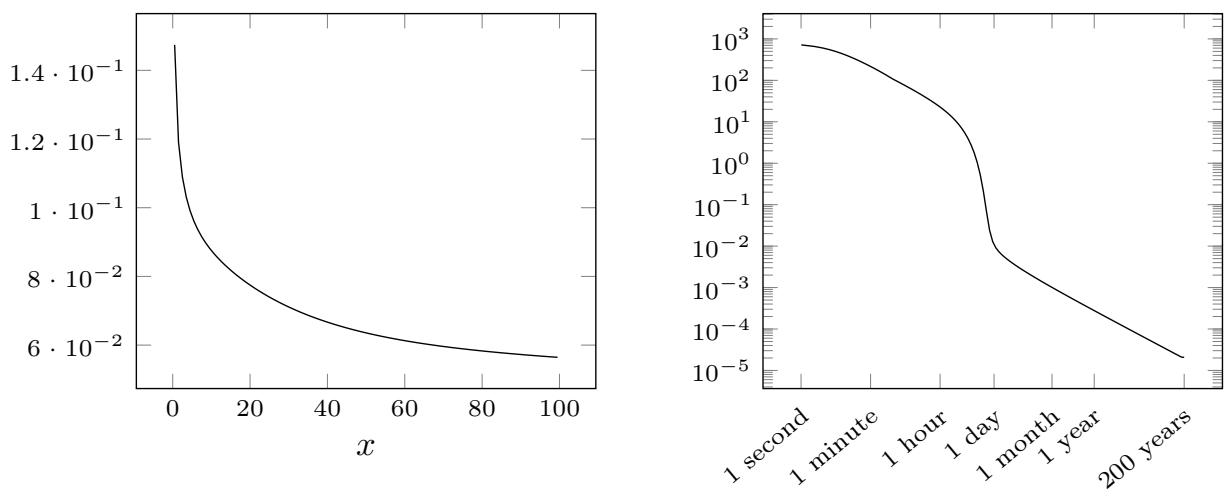

Time

Figure 3 - Value of the Robin coefficient $\beta_{\mathrm{pm}}$ along the interface $\Gamma$ (left) and mean value $\bar{\beta}_{\mathrm{ff}}^{n}=|\Gamma|^{-1} \int_{\Gamma} \beta_{\mathrm{ff}}\left(x, t^{n}\right) \mathrm{d} x$ as a function of time (right).

and to a Neumann boundary condition after the drop of the evaporation rate. It has been checked that for a stopping criterion $\epsilon=10^{-6}$ in (8), the domain decomposition algorithm converges in an average of 3.9 iterations per time step. In practice, one or two iterations are enough to obtain the same solution as the fully coupled algorithm.

Acknowledgement: This work was supported by ANDRA (the french national agency of the radioactive waste management).

\section{References}

[1] R. Masson, L. Trenty, and Y. Zhang, "Formulations of two phase liquid gas compositional Darcy flows with phase transitions," International Journal on Finite Volumes, vol. 11, p. 34, 2014.

[2] A. Lauser, C. Hager, R. Helmig, and B. Wohlmuth, "A new approach for phase transitions in miscible multi-phase flow in porous media," Advances in Water Resources, vol. 34, no. 8, pp. 957 -- 966, 2011.

[3] T. Fetzer, K. Smits, and R. Helmig, "Effect of turbulence and roughness on coupled porous-medium/free-flow exchange processes," Transport in Porous Media, vol. 114, no. 2, pp. 395 -- 424, 2016.

[4] K. Mosthaf, K. Baber, B. Flemisch, R. Helmig, A. Leijnse, I. Rybak, and B. Wohlmuth, "A coupling concept for two-phase compositional porous-medium and single-phase compositional free flow," Water Resources Research, 2011.

[5] R. Masson, L. Trenty, and Y. Zhang, "Coupling compositional liquid gas darcy and free gas flows at porous and free-flow domains interface," Journal of Computational Physics, vol. 321, pp. 708 -- 728, 2016.

[6] C. Japhet, F. Nataf, and F. Rogier, "The optimized order 2 method: Application to convection-diffusion problems," Future Generation Computer Systems, vol. 18, no. 1, pp. 17 -- 30, 2001. 
[7] D. W. Peaceman, Fundamentals of Numerical Reservoir Simulation. Developments in Petroleum Science, Elsevier, 1977.

[8] F. H. Harlow and J. E. Welch, "Numerical calculation of time-dependent viscous incompressible flow of fluid with free surface," Physics of Fluids, vol. 8, no. 12, pp. 2182--2189, 1965. 